\title{
Analytical modelling of soccer heading
}

\author{
ZAHARI TAHA $^{1}$, MOHD HASNUN ARIF HASSAN $^{1, *}$ and \\ ISKANDAR HASANUDDIN ${ }^{2}$
}

\author{
${ }^{1}$ Innovative Manufacturing, Mechatronics \& Sports Laboratory (iMAMS), \\ Faculty of Manufacturing Engineering, Universiti Malaysia Pahang, \\ 26600 Pekan, Pahang, Malaysia \\ ${ }^{2}$ Department of Mechanical Engineering, University of Syiah Kuala, \\ Banda Aceh 32111, Indonesia \\ e-mail: hasnun@ump.edu.my
}

MS received 11 April 2014; revised 22 September 2014; accepted 14 February 2015

\begin{abstract}
Heading occur frequently in soccer games and studies have shown that repetitive heading of the soccer ball could result in degeneration of brain cells and lead to mild traumatic brain injury. This study proposes a two degree-of-freedom linear mathematical model to study the impact of the soccer ball on the brain. The model consists of a mass-spring-damper system, in which the skull, the brain and the soccer ball are modelled as a mass and the neck modelled as a spring-damper system. The proposed model was compared with previous dynamic model for soccer ball-to-head impact. Moreover, it was also validated against drop ball experiment on an instrumented dummy skull and also compared with head acceleration data from previous studies. Comparison shows that our proposed model is capable of describing both the skull and brain accelerations qualitatively and quantitatively. This study shows that a simple linear mathematical model can be useful in giving a preliminary insight on the kinematics of human skull and brain during a ball-to-head impact. The model can be used to investigate the important parameters during soccer heading that affect the brain displacement and acceleration, thus providing better understanding of the mechanics behind it.
\end{abstract}

Keywords. Soccer heading; head impact; brain injury; mathematical model.

\section{Introduction}

Soccer is the most popular sport in the world with a total of 265 million players worldwide, both registered and unregistered. Unfortunately, soccer players can be subjected to several types of injuries including head injury. Soccer players often sustain concussions during game, but a study

*For correspondence 
has shown that more than $70 \%$ of concussed players did not realize that they had suffered concussion (Delaney et al 2002).

Most of the concussions in soccer occur due to head-to-head contact. Other factors include the impact between the head and elbow, knee, ground as well as the ball (Levy et al 2012). The uniqueness of this game is that the players are permitted to use their head to direct the ball during the game, thus causing deliberate impact between the head and the ball. A soccer player can be subjected to six to twelve occasions of heading during a single game (Levy et al 2012; Spiotta et al 2012). In a season, a professional soccer player might perform an average of 800 headings excluding the occasions that take place during trainings.

Many studies have linked purposeful heading in soccer to brain trauma, similar to that found in mild traumatic brain injury (TBI). Both amateur and professional soccer players were evaluated in the past decades in order to prove the theory. The evaluations were done through a series of neuropsychological tests (Tysvaer \& Løchen 1991; Matser et al 1999; Matser et al 2001; Webbe \& Ochs 2003; Witol \& Webbe 2003). These tests assess the neurocognitive performance of the players in several aspects such as planning, memory, attention, visual and verbal. It was found that soccer players had scored poorly in the tests compared with the participants who did not play soccer (Matser et al 1999; Witol \& Webbe 2003). Frequent headers were also found to have obtained even lower scores compared with non-headers (Tysvaer \& Løchen 1991; Matser et al 2001; Webbe \& Ochs 2003). Thus, the number of headings was reported to be inversely proportional to the neurocognitive performance of a soccer player (Matser et al 1999).

Besides the neuropsychological tests, researchers have also used a more advanced method known as diffusion tensor imaging (DTI) in assessing the neurocognitive performance of soccer players. DTI is an advanced magnetic resonance imaging technique that offers detection of microscopic changes in the brain's white matter. It measures the movement of water molecules along the nerve fibres in the brain (axons) that is known as fractional anisotropy (FA) (Lipton et al 2013). Researchers have attempted to assess both amateur and professional soccer players using this technique (Koerte \& Ertl-Wagner 2012; Lipton et al 2013). These studies have found abnormalities in the white matter of the frequent headers that were similarly found in patients with mild TBI. In addition, the former study found compelling evidence that exceeding the threshold level of 885 to 1,550 headings a year will result in significantly lower FA values that is associated with cognitive impairment in patients with TBI.

Moreover, a recent study has attempted a different approach, in which a tablet-based application was developed and used to evaluate the cognitive effect of soccer ball heading (Zhang et al 2013). Results obtained have revealed that soccer players were significantly slower than control group in voluntary response task, which suggests that soccer ball heading can lead to alterations of cognitive function that are similar to mild TBI. This study introduces an easier method in assessing the cognitive functions that can be applied not only to soccer players, but also to athletes involve in other contact sports.

In actuality, a single heading does not pose threat to the player since it does not cause head acceleration capable of causing brain injury. Heading a soccer ball results in head acceleration of less than $10 \mathrm{~g}$, which is lower than the threshold of sport-related concussions of 40-60 g (McCrory 2003). Nonetheless, previous studies have shown convincing evidence that repetitive heading in soccer might affect the neurocognitive performance of a soccer player and could cause abnormalities in the brain, which could then lead to brain trauma.

Researchers have attempted to experimentally measure head acceleration endured by soccer players during heading. The subjects used in the studies include human volunteers (Bayly et al 2002; Naunheim et al 2003a) and dummy head form (Naunheim et al 2003b; Withnall et al 2005). In studies involving human subjects, a series of accelerometers were attached to 
the subjects' head. From the data obtained, relative motion equation for rigid body was used to calculate the acceleration of the centre of gravity of the head. These studies however produced the acceleration of the head as a single unit. The concern of possible brain injury in soccer is in fact associated with the acceleration of the brain. Thus, it is vital to quantify the acceleration of the brain during soccer heading.

In estimating the acceleration of the brain during soccer heading, modelling and simulation is definitely the optimal approach. Measuring brain acceleration in vivo is almost impossible, thus researchers have tried to simulate heading manoeuvre through mathematical models. Naunheim et al developed a model to validate the experiment conducted on measuring head acceleration during soccer heading, but the model neglected the contribution of neck musculature (Naunheim et al 2003a). An improved model was then proposed that took into account neck musculature (Riches 2006). Both models generate linear and angular acceleration of the head upon soccer ball impact. But these models do not have the capability to assess the acceleration of skull and brain individually. Brain injury occurs due to the acceleration of the brain, thus it is essential to estimate the brain and skull acceleration separately rather than assessing acceleration of the head as a single unit.

This study proposes a model that provides linear acceleration of both skull and brain during soccer ball impact individually. The model produces the relationship between the velocity of soccer ball before impact and the resultant linear accelerations of the skull and brain. Data obtained for skull accelerations were compared with published experimental data of head accelerations during soccer heading (Bayly et al 2002; Naunheim et al 2003a; Shewchenko et al 2005; Withnall et al 2005). In addition, the brain accelerations were validated with an experiment performed on an instrumented dummy skull.

\section{Methods}

\subsection{Mathematical model}

It is assumed that a ball that travels with a defined velocity impacts the skull that is represented by a mass $m_{1}$. It is attached to a spring (stiffness, $k_{1}$ ) and a dashpot (damping coefficient, $c_{1}$ ) that represent the neck. Another mass $m_{2}$, which denotes the brain, is attached to the skull by a spring-damper system (stiffness, $k_{2}$ and damping coefficient, $c_{2}$ ) that represents its suspension characteristics. Figure 1(a) depicts the two-degree-of-freedom linear mathematical model, whilst figure 1(b) shows the free-body diagram of the proposed model.

The mass of the skull is taken as $m_{1}=3.5 \mathrm{~kg}$, whereas the mass of the brain is defined as $m_{2}=1.5 \mathrm{~kg}$. (Dirisala et al 2012) have proposed several values for both the stiffness $(k=1.8$, 18 and $1,800 \mathrm{kN} / \mathrm{m})$ and damping coefficient $(c=0,450$ and 1,800 Ns/m) of the neck. Through parametric analyses, we have found that the neck's stiffness of $k_{1}=1,800 \mathrm{kN} / \mathrm{m}$ and the damping coefficient of $c_{1}=450 \mathrm{Ns} / \mathrm{m}$ suit our model best and therefore were used in the model. As for the brain, (Baghaei et al 2009) have performed curve fitting on published experimental data and both the stiffness and the damping coefficient of the brain were obtained as $k_{2}=156 \mathrm{kN} / \mathrm{m}$ and $c_{2}=340 \mathrm{Ns} / \mathrm{m}$, respectively. These values were used in our model to define the suspension characteristics of the brain.

The accelerations of the skull and the brain are given by the following equations:

$$
\begin{gathered}
m_{1} \ddot{x}_{1}=m_{1} g-k_{1} x_{1}-c_{1} \dot{x}_{1}+k_{2}\left(x_{2}-x_{1}\right)+c_{2}\left(\dot{x}_{2}-\dot{x}_{1}\right)+m_{b} g+k_{b} x_{b}+c_{b} \dot{x}_{b 1} \\
m_{2} \ddot{x}_{2}=m_{2} g-k_{2}\left(x_{2}-x_{1}\right)-c_{2}\left(\dot{x}_{2}-\dot{x}_{1}\right) .
\end{gathered}
$$




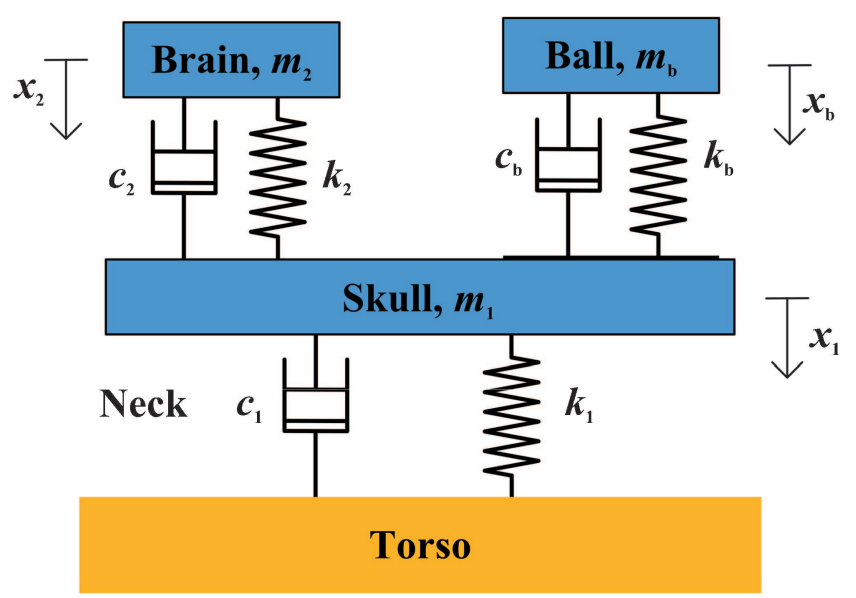

(a)

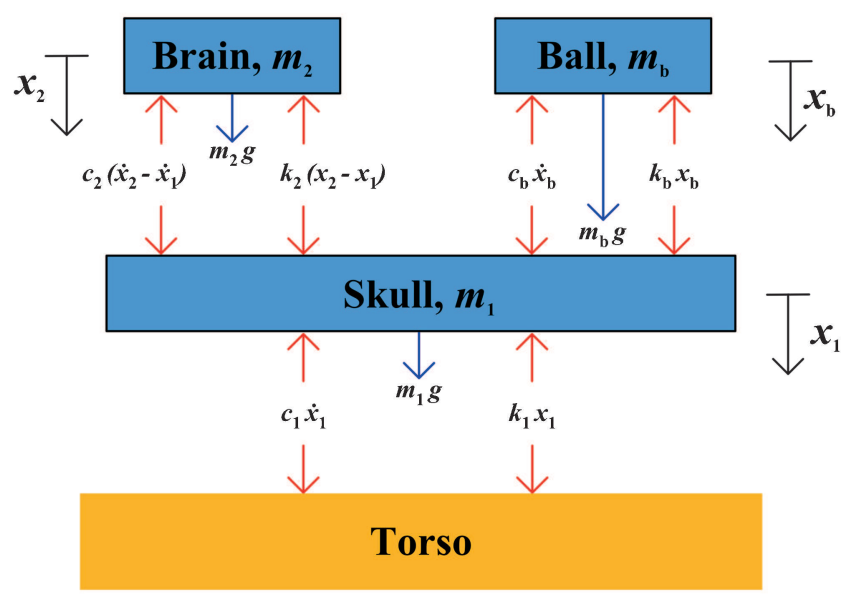

(b)

Figure 1. (a) Proposed mathematical model of soccer ball-to-head impact, (b) free-body diagram of the proposed model.

$\ddot{x}_{1}$ and $\ddot{x}_{2}$ represent the acceleration of the skull and brain, respectively, $g$ represents the gravitational acceleration, whilst $\dot{x}_{1}$ and $\dot{x}_{2}$ are the velocity of the skull and brain, respectively. In addition, $x_{1}$ and $x_{2}$ denote the displacement of the skull and brain, respectively. The mass of the skull, brain and ball is denoted by $m_{1}, m_{2}$ and $m_{\mathrm{b}}$, respectively. It is assumed that the initial velocity of the skull $\dot{x}_{1}^{\prime}$ is defined by the equation of the conservation of linear momentum (Hibbeler 2009):

$$
\dot{x}_{1}^{\prime}=\frac{m_{b}\left(\dot{x}_{b 1}-\dot{x}_{b 2}\right)}{m_{1}},
$$

where $\dot{x}_{b 1}$ and $\dot{x}_{b 2}$ are the inbound and rebound velocity of the soccer ball, respectively.

The last three terms on the right hand side of Eq. (1) represent the force exerted on the skull by the soccer ball as it is assumed to behave as a mass-spring-damper system (Nagurka \& Huang 2006). The mass of the ball $m_{\mathrm{b}}$ is taken as $0.45 \mathrm{~kg}$ whilst the stiffness of the ball $k_{\mathrm{b}}$, its 
maximum deformation during impact $x_{\mathrm{b}}$, its damping coefficient $c_{\mathrm{b}}$ and the velocity of the ball before impact $\dot{x}_{b 1}$ were experimentally determined.

A drop ball experiment was conducted to obtain the aforementioned parameters. A soccer ball was inflated to the manufacturer's recommended air pressure of 0.7 bars and it was dropped from the height of $0.5,1.0,1.5$ and $2.0 \mathrm{~m}$ onto a flat rigid surface. A high-speed camera (model SV643C, EPIX Inc.) was used to capture the sequence of images of the motion. The camera was placed on the flat surface parallel to the dropping position of the ball. The camera was operated using XCAP software (version 3.7, EPIX, Inc.) and set to record 1,000 frames per second. From the recorded images, the velocities of the ball before and after impact were determined and the coefficient of restitution (COR) was calculated alongside the duration of impact. Table 1 shows the parameters that have been measured in the experiment (inbound and rebound velocity, and contact time) and have been calculated based on the data obtained (COR, stiffness and damping coefficient).

From the duration of impact $\Delta T$ obtained from the recorded images and the calculated coefficient of restitution $e$, the stiffness $k_{\mathrm{b}}$ and damping coefficient $c_{\mathrm{b}}$ of the soccer ball were determined using the following equations (Nagurka \& Huang 2006):

$$
\begin{gathered}
k_{b}=\frac{m_{b}}{(\Delta T)^{2}}\left[\pi^{2}+(\ln e)^{2}\right] \\
c_{b}=-\frac{2 m_{b}}{\Delta T} \ln e .
\end{gathered}
$$

The values of $k_{\mathrm{b}}=36,833 \mathrm{~N} / \mathrm{m}, c_{\mathrm{b}}=15.17 \mathrm{Ns} / \mathrm{m}, e=0.8307$ and $\Delta T=0.012 \mathrm{~s}$ were obtained and assumed constant regardless of the velocity of the ball. On the other hand, the maximum ball deformation during impact varies according to the inbound velocity of the ball. Therefore, the maximum ball deformation was determined using the following equation (Nagurka \& Huang 2006):

$$
x_{b}=-\frac{\dot{x}_{b 1}}{\omega_{d}} \exp \left(-\frac{c_{b}}{2 m_{b}} \cdot \frac{\Delta T}{2}\right) \sin \left(\omega_{d} \frac{\Delta T}{2}\right),
$$

where $\omega_{\mathrm{d}}$ is the damped natural frequency and is defined as follows:

$$
\omega_{d}=\frac{1}{2 m_{b}} \sqrt{4 k_{b} m_{b}-c_{b}^{2}}
$$

All parameters involved were defined in the model, and Eqs. (1) and (2) were solved for $\ddot{x}_{1}$ and $\ddot{x}_{2}$ using Euler method with a step size of 0.0001 .

Table 1. Several parameters of a soccer ball determined from the drop ball experiment.

\begin{tabular}{lcccccc}
\hline $\begin{array}{l}\text { Drop } \\
\text { height }(\mathrm{m})\end{array}$ & $\begin{array}{c}\text { Inbound velocity, } \\
\dot{x}_{b 1}(\mathrm{~m} / \mathrm{s})\end{array}$ & $\begin{array}{c}\text { Rebound velocity, } \\
\dot{x}_{b 2}(\mathrm{~m} / \mathrm{s})\end{array}$ & $\begin{array}{c}\text { Contact time, } \\
\Delta T(\mathrm{~s})\end{array}$ & $\mathrm{COR}, e$ & $\begin{array}{c}\text { Stiffness, } \\
k\end{array}$ & $\begin{array}{c}\text { Damping } \\
\text { coefficient, } c\end{array}$ \\
\hline 0.5 & 2.931 & 2.443 & 0.012 & 0.8333 & 36829 & 14.92 \\
1.0 & 3.927 & 3.273 & 0.012 & 0.8331 & 36829 & 14.94 \\
1.5 & 4.829 & 4.015 & 0.012 & 0.8314 & 36832 & 15.11 \\
2.0 & 5.546 & 4.577 & 0.012 & 0.8252 & 36842 & 15.72 \\
2.3 & 5.985 & 4.970 & 0.012 & 0.8305 & 36833 & 15.20 \\
& Average & & 0.012 & 0.8307 & 36833 & 15.17 \\
\hline
\end{tabular}




\subsection{Experimental validation}

In order to validate our mathematical model, we have performed a drop ball experiment on an instrumented dummy skull. The dummy skull is made of acrylonitrile butadiene styrene (ABS) and it was fabricated through rapid prototyping technique. The inner cavity of the skull was filled with ultrasound gel (type EcoGel 200, Eco-Med Pharmaceutical Inc.) that represents the brain. The gel was selected due to its density $\left(1.01 \mathrm{~kg} / \mathrm{m}^{3}\right)$ that is close to that of the human brain $(1.04$ $\mathrm{kg} / \mathrm{m}^{3}$ ) (Dirisala et al 2012). To measure the acceleration of the gel, a tri-axial accelerometer (model 356A67, PCB Piezoelectronics) was placed inside the gel approximately at the centre of gravity of the skull ( $7 \mathrm{~cm}$ from the foramen magnum). It is assumed that the accelerations obtained from the accelerometer represent the brain acceleration.

A string was attached to a standard size-5 soccer ball (model Premier League Strike, Nike, Inc.) through one of its stitches. The string was wrapped around a pulley and the ball was dropped onto the instrumented dummy skull from a height of $2.2 \mathrm{~m}$ as shown in figure 2 . The chosen height is the highest possible height in the laboratory where the experiment was conducted. The ball was dropped for 30 times and the average peak acceleration of the gel was determined.

The accelerometer was connected to a data acquisition (DAQ) module (model NI 9234, National Instruments) that was attached to a compact DAQ chassis (model NI cDAQ-9171, National Instruments). The signal from accelerometer was read using DASYLab software (version 10, National Instruments). The analogue signals were low-pass filtered $(1,000 \mathrm{~Hz}$, Butterworth) and were sampled at 5,000 Hz. A high-speed camera (model SV643C, Epix Inc.) was used to capture the motion of the soccer ball to determine its inbound and rebound velocity as well as the duration of impact.

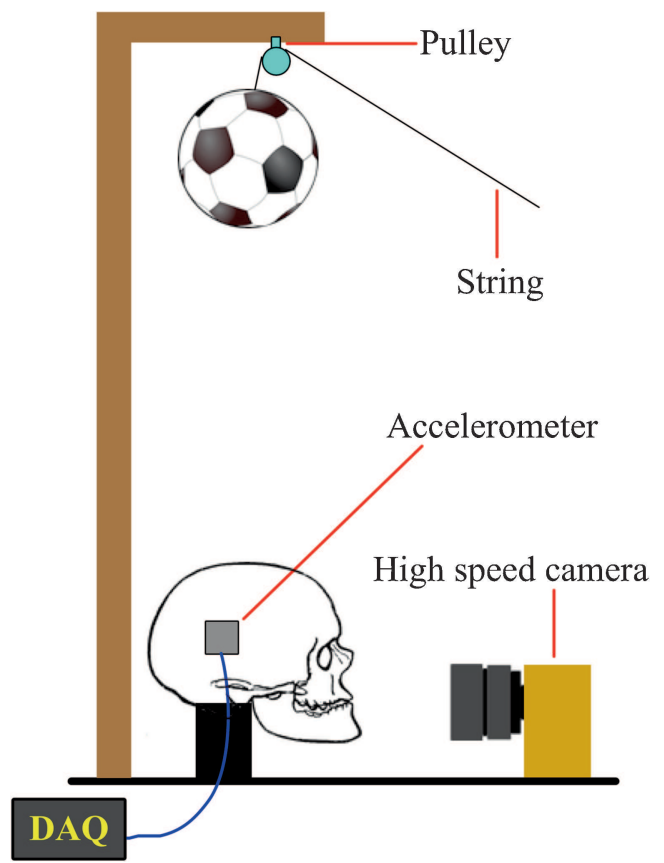

Figure 2. Dropped-ball test on dummy skull. 


\subsection{Comparison with previous studies}

We have also validated our model against head acceleration data obtained from published literatures (Bayly et al 2002; Naunheim et al 2003a; Shewchenko et al 2005; Withnall et al 2005). They have performed soccer heading experiments on both human subjects and surrogates. They have measured head accelerations during heading using a series of accelerometers. For this purpose, we have reduced the impact duration, $\Delta T$ from $0.012 \mathrm{~s}$ to $0.01 \mathrm{~s}$ to obtain the best agreement. Other parameters remained as previously defined.

\section{Results and discussion}

A two-degree of freedom linear mathematical model was developed to predict the skull and brain accelerations during an impact with a soccer ball. The important components of the model, which include the impact force, skull and brain accelerations, and brain's stiffness and damping components as well as their displacements in the case of a drop height of $2.2 \mathrm{~m}$, which corresponds to the ball's inbound velocity of $5.94 \mathrm{~m} / \mathrm{s}$ are plotted in figure $3 \mathrm{a}, 3 \mathrm{~b}$ and $3 \mathrm{c}$, respectively.

Initially after impact, the neck compresses, thus causing the skull to accelerate downward. The movement of the skull results in the acceleration of the brain in the same direction. As the velocity and displacement of the brain increase, the motion is resisted by the brain's damping, and then followed by the brain's stiffness as shown in figure $3 \mathrm{~b}$.

As the impact force approaches its maximum value (maximum ball deformation), the skull also approaches its peak downward acceleration. The skull starts to accelerate upward as it reaches its peak downward acceleration, whereas the brain is still accelerating downward (figure 3a). The brain's damping resistance starts to decrease, whilst the brain's stiffness continues resisting the motion (figure $3 \mathrm{~b}$ ). At the time $t=0.0067 \mathrm{~s}$, the skull reaches its peak displacement as depicted in figure 3c. After reaching its peak displacement, the skull starts to move in the opposite direction whilst the brain is still moving upward until it reaches its peak displacement at $t=0.0085 \mathrm{~s}$. As shown in figure 3c, the skull has recorded a peak displacement of $0.553 \mathrm{~mm}$, whilst the brain's peak displacement is $0.892 \mathrm{~mm}$. This suggests that during this particular time, part of the brain (depending on the location of impact) will collide with the inner wall of the skull. This collision will probably result in brain trauma.

During the first phase of the skull and brain motion, the peak downward brain acceleration is $10 \%$ higher than that of the skull for the defined ball velocity of $5.94 \mathrm{~m} / \mathrm{s}$, although it occurs a few milliseconds later. It is observed that the difference between peak downward skull acceleration and peak downward brain acceleration decreases with increasing ball velocity. As the impact force lessens, the skull starts accelerating in the upward direction. At the instant the ball leaves the head as denoted by the impact force $=0 \mathrm{~N}$, the acceleration of the skull equals zero (figure $3 a)$, the brain's damping component equals zero whilst the brain's stiffness component is at a maximum (figure 3b). Our results concur with the data from previous model (Riches 2006).

After the ball leaves the head, the skull continues to accelerate in upward direction (figure $3 \mathrm{a}$ ). The brain also accelerates upward and reaches its maximum a few milliseconds after the skull reaches its peak upward acceleration as shown in figure 3a. The brain's damping component dominates the mechanics to ease the subsequent oscillations (figure $3 b$ ). Peak skull acceleration occurs within the first $15 \mathrm{~ms}$ after the soccer ball impact. This agrees with the published experimental data (Naunheim et al 2003a; Shewchenko et al 2005). In the second phase of the motion, the peak skull acceleration is higher than that of the brain. It takes approximately $60-70 \mathrm{~ms}$ for the skull and brain to completely stop accelerating. The duration of oscillation obtained from our 


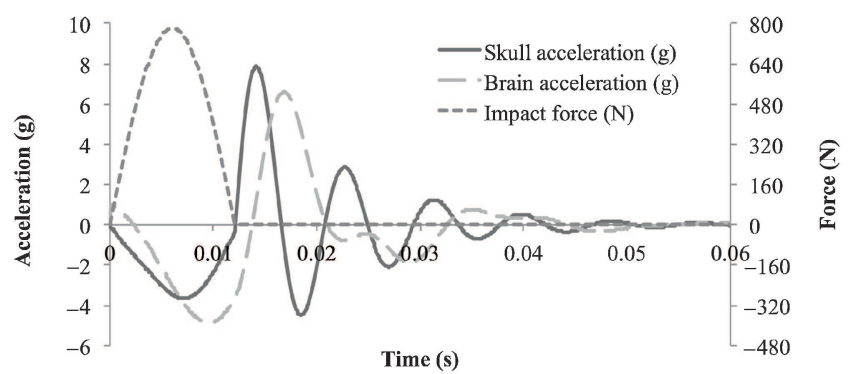

(a)

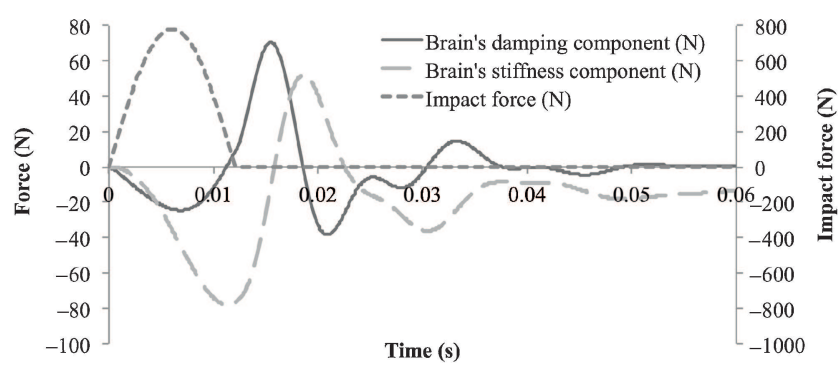

(b)

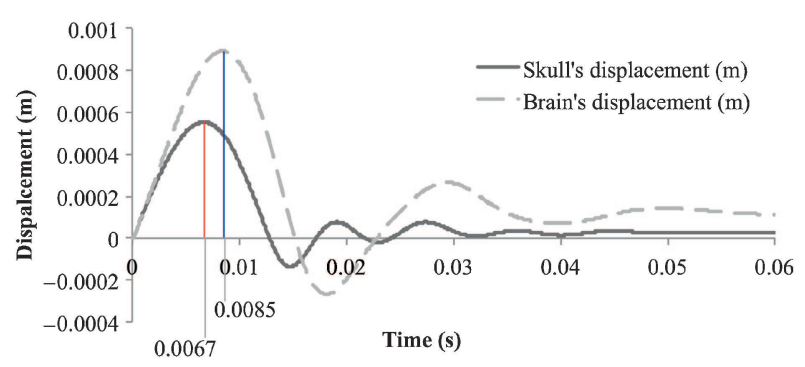

(c)

Figure 3. (a) Impact force, skull and brain acceleration, (b) brain's damping and stiffness component, (c) skull's and brain's displacement for the drop height of $2.2 \mathrm{~m}, \dot{x}_{b}=5.94 \mathrm{~m} / \mathrm{s}, \Delta T=0.012 \mathrm{~s}$.

model agrees with previous linear acceleration data obtained from published experimental data (Naunheim et al 2003a; Shewchenko et al 2005).

We have also analysed the influence of neck's stiffness and damping coefficient on the skull and brain acceleration during impact with soccer ball. For this purpose, the stiffness and damping coefficient of the neck were individually varied, whilst the other parameter remains constant as shown in figure 4a and 4b. It is clear from figure 4a that lower neck's stiffness results in higher skull and brain acceleration, and as neck's stiffness increases, brain acceleration decreases. On the other hand, neck's damping coefficient has little effect of the skull and brain acceleration. Increasing neck's damping coefficient only results in slight decrement of skull and brain acceleration. Neck's stiffness represents the strength of neck muscle. Thus, the amount of skull and brain acceleration might vary between genders, age or skill's level of the player. Our findings support previous experimental results, which show that female soccer players exhibited higher head acceleration during heading compared with male players (Tierney et al 2008). 


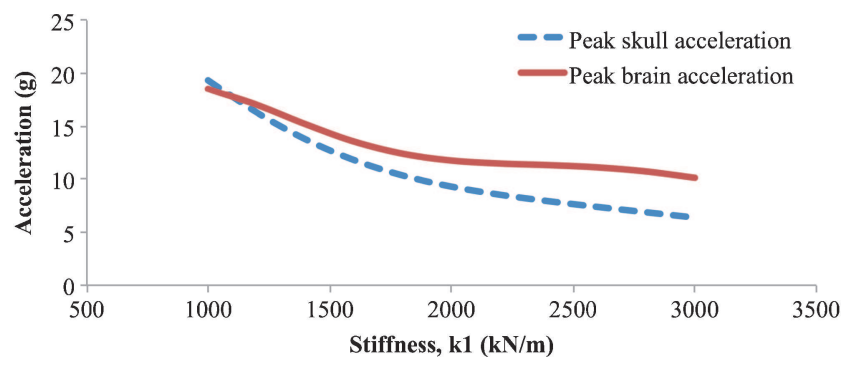

(a)

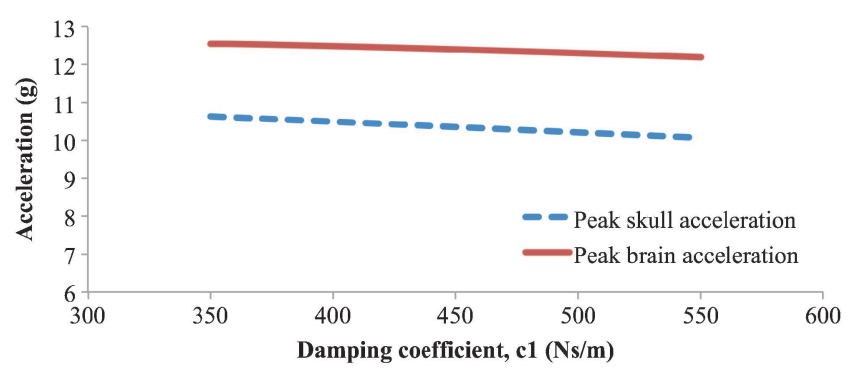

(b)

Figure 4. (a) Acceleration against stiffness, $\mathrm{k}_{1}$ with $\mathrm{c}_{1}=350 \mathrm{Ns} / \mathrm{m}$. (b) Acceleration against damping coefficient, $\mathrm{c}_{1}$ with $\mathrm{k}_{1}=1,800 \mathrm{kN} / \mathrm{m}$.

\subsection{Model validation}

To further validate our model, a drop ball test was conducted on an instrumented dummy skull. A soccer ball was dropped from $2.2 \mathrm{~m}$ height (the highest possible drop height in the laboratory where the experiment was conducted) onto the dummy skull for 30 times $(n=30)$. The resultant acceleration was determined from the acceleration data obtained from each axis of the accelerometer. Figure 5 shows the gel's acceleration recorded in the drop ball test, which is assumed to represent the brain's acceleration. Table 2 shows the comparison between the acceleration of the gel obtained from the experiment and the brain acceleration obtained from the model for the same velocity of the ball before impact. The result shows that peak linear acceleration of

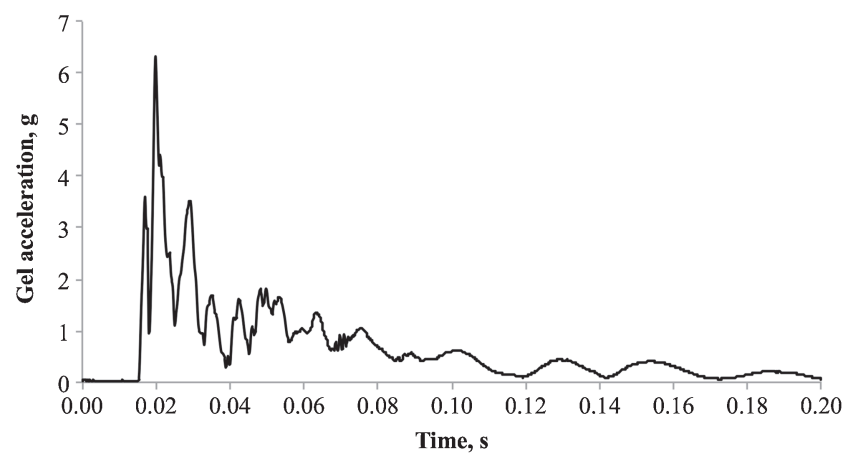

Figure 5. Gel acceleration obtained from the drop ball test. 
Table 2. Means ( \pm SD) of peak brain acceleration from experiment and model.

\begin{tabular}{lcccr}
\hline \multirow{2}{*}{ Drop height, $\mathrm{m}$} & Ball velocity, $\mathrm{m} / \mathrm{s}$ & \multicolumn{2}{c}{ Peak brain acceleration, $\mathrm{g}$} & \\
\cline { 3 - 4 } & 5.94 & Experiment & Model & Difference \\
\hline 2.2 & $6.375 \pm 0.836$ & 6.582 & $4.35 \%$ \\
\hline
\end{tabular}

the brain produced by our model agrees with the peak resultant linear acceleration of the gel obtained from experiment with a difference of less than $5 \%$.

Furthermore, we have also compared the peak head acceleration data from previous studies with the peak skull acceleration produced by our model as shown in table 3 . It is observed that the model has produced peak acceleration data that are in good agreement with literature except for some values. Comparison with study by (Naunheim et al 2003b) for ball speed of $15 \mathrm{~m} / \mathrm{s}$ shows a difference of $11.89 \%$. Since the standard deviation for this value is $1.51 \mathrm{~g}$, subtracting this value from the acceleration value decreases the difference to only $7.3 \%$. This also applies to the data from (Withnall et al 2005) for ball speed of $10 \mathrm{~m} / \mathrm{s}$. By adding the standard deviation value of $0.63 \mathrm{~g}$ to the data, the resulting difference becomes $9.1 \%$. Another data that shows a large difference is from (Shewchenko et al 2005) for ball speed of $7.6 \mathrm{~m} / \mathrm{s}$. However, they have stated that the standard deviation for the ball speed is $1.3 \mathrm{~m} / \mathrm{s}$. Thus, if we defined the ball speed as $8.9 \mathrm{~m} / \mathrm{s}$ in our model, the resulting peak skull acceleration is $15.84 \mathrm{~g}$, that is $1.5 \%$ difference from the literature value.

It is evident that our model is capable of predicting skull and brain acceleration during soccer ball impact. Our model shows that upon impact with the ball, the skull accelerates in the direction of the impact force, whilst the brain takes a few milliseconds before it starts to accelerate in the same direction. After reaching its first peak acceleration, the skull starts to accelerate in the opposite direction, whilst the brain is still accelerating in the previous direction. At one point, the skull and brain collides due to the phase difference in the oscillation of both body. This collision might result in brain trauma, but further study is needed to prove our findings. We have also demonstrated that the neck's stiffness has more influence on the skull and brain acceleration compared with its damping coefficient. Stronger neck muscle is required when heading soccer ball to avoid brain trauma. Hence, individuals with weak neck muscle such as children or female players should avoid from frequent soccer ball heading.

Table 3. Comparison between literature values and proposed model ( \pm SD).

\begin{tabular}{lcccc}
\hline \multirow{2}{*}{ Author (year) } & & \multicolumn{2}{c}{ Peak head acceleration $(\mathrm{g})$} & \\
\cline { 3 - 4 } & Ball speed $(\mathrm{m} / \mathrm{s})$ & Literature & Model & Difference (\%) \\
\hline (Bayly et al 2002) & 9 & $16.1 \pm 1.0$ & 16.02 & 0.51 \\
(Naunheim et al 2003b) & 12 & $20.3 \pm 2.8$ & 21.41 & 5.45 \\
& 9 & $15.11 \pm 0.70$ & 16.02 & 6.01 \\
(Shewchenko et al 2005) & 12 & $21.31 \pm 1.78$ & 21.41 & 0.46 \\
& 15 & $30.41 \pm 1.51$ & 26.80 & 11.89 \\
(Withnall et al 2005) & $7.6 \pm 1.3$ & 15.6 & 13.50 & 13.44 \\
Hybrid III Dummy Headform & $10.1 \pm 1.1$ & 17.43 & 17.99 & 3.24 \\
& 20 & $15.7 \pm 0.63$ & 17.81 & 13.47 \\
& 30 & 32.9 & 35.78 & 8.74 \\
& & $55.4 \pm 2.4$ & 53.74 & 3.00 \\
\hline
\end{tabular}




\section{Conclusion}

A two-degree of freedom linear mathematical model has been developed to predict the skull and brain accelerations during impact with soccer ball. The model takes into account the neck muscle properties and produces individual kinematics data for skull and brain. Parameters involved in the model were defined according to literature values. The peak skull acceleration occurs just after the ball leaves the head, whilst the peak brain acceleration takes place a few milliseconds afterwards. Our model shows that the relative displacement of the brain with respect to the skull might, at one point, cause a collision between these two components. This collision could result in brain trauma, but further investigation is needed to prove this finding. It was also found that stronger neck muscle is required to prevent brain injury during soccer heading. The model serves as an initial stage in finding the suitable material that can dissipate the impact force caused by soccer heading.

\section{References}

Baghaei S M, Sadegh A M and Rajaai S M 2009 A mathematical head/brain model for investigation of damping characteristics of SAS in low velocity head impacts. In: 25th Southern biomedical engineering conference 2009, 15-17 May 2009. Miami, Florida, USA

Bayly P V, Naunheim R, Standeven J, Neubauer J S, Lewis L and Genin G M 2002 Linear and angular accelerations of the human head during heading of a soccer ball. In: Engineering in Medicine and Biology, 2002. 24th annual conference and the annual fall meeting of the Biomedical Engineering Society EMBS/BMES conference, 2002. Proceedings of the second joint 3: 2577-2578 vol. 3. doi: 10.1109/IEMBS.2002.1053434

Delaney J S, Lacroix V J, Leclerc Suzanne and Johnston K M 2002 Concussions among university football and soccer players. Clin. J. Sport Med. 12: 331-338

Dirisala V, Karami G and Ziejewski M 2012 Effects of neck damping properties on brain response under impact loading. Int. J. Numer. Methods Biomed. Eng.472-494 doi:10.1002/cnm

Hibbeler R C 2009 Engineering mechanics: Dynamics in SI units pack. Prentice Hall

Koerte I K and Ertl-Wagner B 2012 White matter integrity in the brains of professional soccer players without a symptomatic concussion. J. Am. Med. Assoc. 308: 2006-2008

Levy Michael L, Aimen S Kasasbeh, Lissa Catherine Baird, Chiazo Amene, Jeff Skeen and Larry Marshall 2012 Concussions in soccer: A current understanding. World Neurosurg. 78: 535-44 doi: 10.1016/j.wneu.2011.10.032

Lipton M L, Kim N and Zimmerman M E 2013 Soccer heading is associated with white matter microstructural and cognitive abnormalities. Radiology 268: 850-857

Matser E J T, Kessels A G, Lezak M D, Jordan B D and Jaap Troost 1999 Neuropsychological impairment in amateur soccer players. J. Am. Med. Assoc. 282: 971-973

Matser J T, Kessels A G, Lezak M D and Troost J 2001 A dose-response relation of headers and concussions with cognitive impairment in professional soccer players. J. Clin. Exper. Neuropsychol. 23: 770-4 doi: 10.1076/jcen.23.6.770.1029

McCrory P R 2003 Brain injury and heading in soccer. Br. Med. J. 327(7411): 351-352

Nagurka Mark and Shuguang Huang 2006 A mass-spring-damper model of a bouncing ball. Int. J. Eng. Educ. 22: 9

Naunheim Rosanne S, Philip V Bayly, John Standeven, Jeremy S Neubauer, Larry M Lewis and Guy M Genin 2003a Linear and angular head accelerations during heading of a soccer ball. Med. Sci. Sports Exer. 35: 1406-1412 doi:10.1249/01.MSS.0000078933.84527.AE

Naunheim Rosanne S, Amanda Ryden, John Standeven, Guy Genin, Larry Lewis, Paul Thompson and Phil Bayly 2003b Does soccer headgear attenuate the impact when heading a soccer ball? Acad. Emerg. Med.: Official J. Soc. Acad. Emerg. Med. 10: 85-90 
Riches Philip E 2006 A dynamic model of the head acceleration associated with heading a soccer ball. Sports Eng. 9: 39-47 doi:10.1007/BF02844261

Shewchenko N, Withnall C, Keown M, Gittens R and Dvorak J 2005 Heading in football. Part 2: Biomechanics of ball heading and head response. Br. J. Sports Med. 39(Suppl 1): i26-32 doi: 10.1136/bjsm.2005.019042

Spiotta Alejandro M, Adam J Bartsch and Edward C Benzel 2012 Heading in soccer: Dangerous play? Neurosurgery 70 : 1-11

Tierney Ryan T, Michael Higgins, Shane V Caswell, Jessica Brady, Krista McHardy, Jeffrey B Driban and Kurosh Darvish 2008 Sex differences in head acceleration during heading while wearing soccer headgear. J. Athletic Training 43: 578-84 doi:10.4085/1062-6050-43.6.578

Tysvaer A T and Løchen E A 1991 Soccer injuries to the brain: A neuropsychologic study of former soccer players. Am. J. Sports Med. 19: 56-60

Webbe Frank M and Shelley R Ochs 2003 Recency and frequency of soccer heading interact to decrease neurocognitive performance. Appl. Neuropsychol. 10: 31-41 doi:10.1207/S15324826AN1001_5

Withnall C, Shewchenko N, Wonnacott M and Dvorak J 2005 Effectiveness of headgear in football. Br. J. Sports Med. 39(Suppl 1): i40-i48; discussion i48 doi:10.1136/bjsm.2005.019174

Witol Adrienne D and Frank M Webbe 2003 Soccer heading frequency predicts neuropsychological deficits. Arch. Clin. Neuropsychol.: Official J. Natl. Acad. Neuropsychol. 18: 397-417

Zhang Marsha R, Stuart D Red, Angela H Lin, Saumil S Patel and Anne B Sereno 2013 Evidence of cognitive dysfunction after soccer playing with ball heading using a novel tablet-based approach. PloS one 8: e57364 doi:10.1371/journal.pone.0057364 